\title{
Aortic intimointimal intussusception in chronic type B dissection causing dynamic obstruction of visceral arteries
}

Sang Yoon Kim, MD, ${ }^{a}$ Ho Young Hwang, MD, PhD, ${ }^{a}$ Whal Lee, MD, PhD, ${ }^{b}$ and Hyuk Ahn, MD, PhD, ${ }^{a}$ Seoul, Korea

See related commentary on pages e35-6.

From the ${ }^{\mathrm{a}}$ Department of Thoracic and Cardiovascular Surgery, Seoul National University Hospital, Seoul, Korea; and ${ }^{\mathrm{b}}$ Department of Radiology, Seoul National University Hospital, Seoul, Korea.

Disclosures: Authors have nothing to disclose with regard to commercial support. Received for publication Nov 30, 2014; revisions received April 15, 2015; accepted for publication May 9, 2015; available ahead of print June 11, 2015.

Address for reprints: Hyuk Ahn, MD, PhD, Department of Thoracic and Cardiovascular Surgery, Seoul National University Hospital, 101 Daehak-ro, Jongno-gu, Seoul 110-744, Korea (E-mail: ahnhyuk@ @nu.ac.kr).

J Thorac Cardiovasc Surg 2015;150:e33-5 $0022-5223 / \$ 36.00$

Copyright $\odot 2015$ by The American Association for Thoracic Surgery http://dx.doi.org/10.1016/j.jtcvs.2015.05.033
Aortic intimointimal intussusception, first reported in 1962 , is a rare form of aortic dissection with a circumferential intimal tear and a flap inverted into the internal space. ${ }^{1}$ Aortic intimointimal intussusception has been reported in several cases of acute dissection. We describe the preoperative and intraoperative findings of aortic intimointimal intussusception in a chronic type B dissection that caused dynamic obstruction of the abdominal organs.

\section{CLINICAL SUMMARY}

A 38-year-old man visited a local hospital with intermittent left flank and upper abdominal pain. The patient's medical history included blunt trauma to his back 8 years earlier. Despite the traumatic event, he did not receive a diagnostic
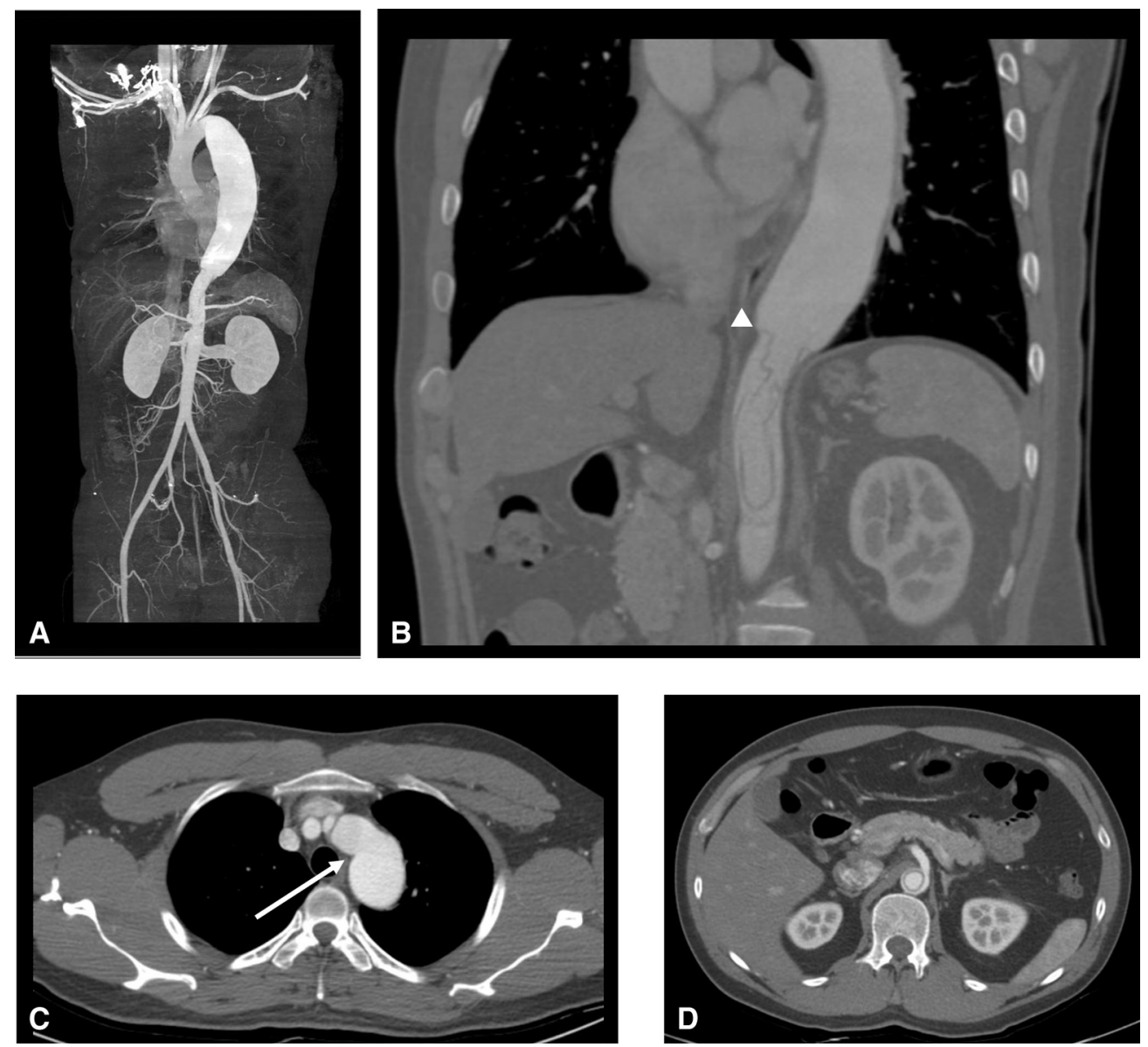

FIGURE 1. Preoperative CT aortic intimointimal intussusception image. A, Three-dimensional reconstruction. B, Inverted flap on reconstructed coronal image, including hinge point (arrowhead) at the level of diaphragm. C, Abrupt aneurysmal change of proximal descending thoracic aorta indicates the suspected intimal tear site (arrow). D, Suspected dynamic obstruction of celiac axis on axial image. 

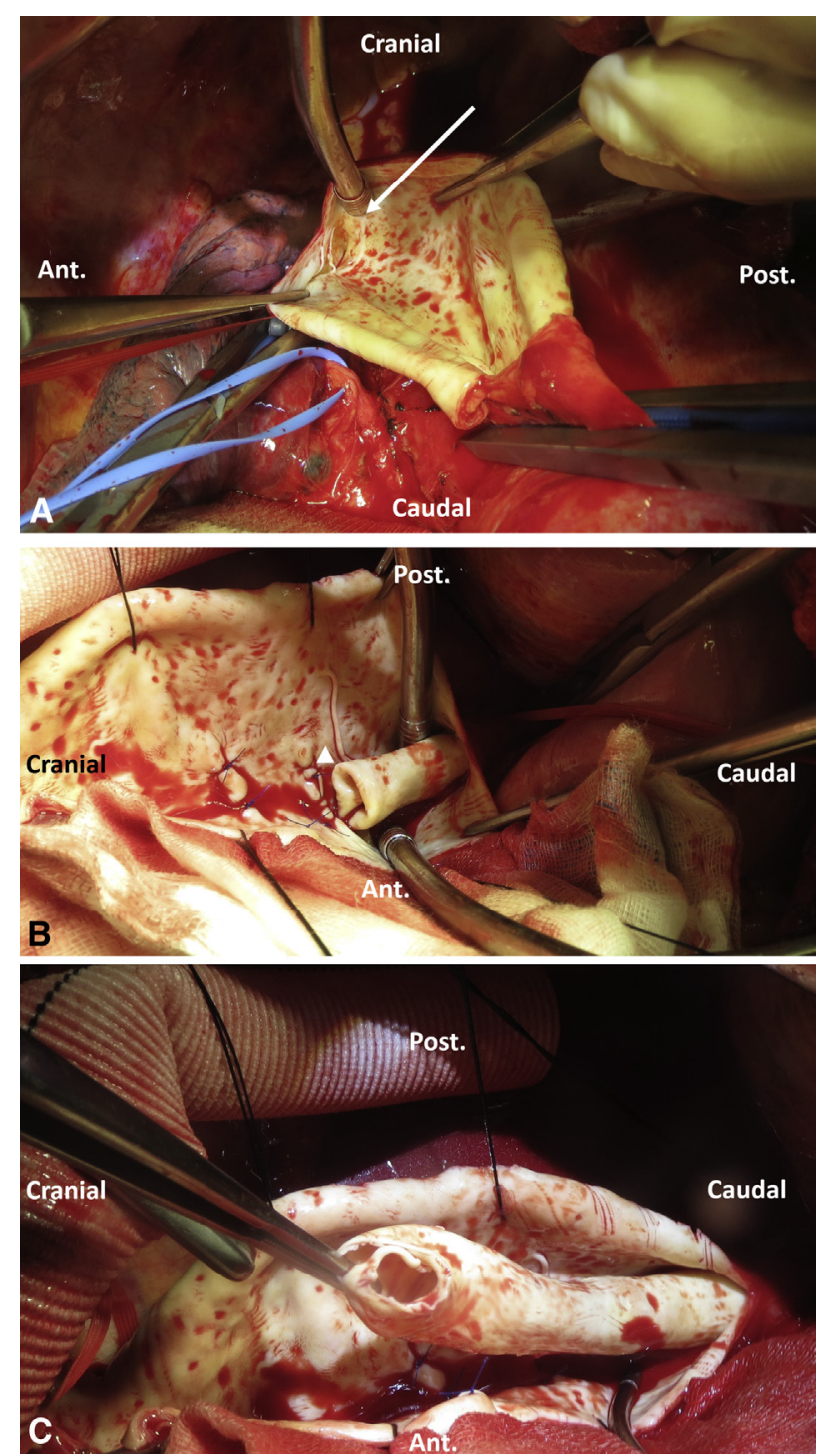

FIGURE 2. Operative aortic intimointimal intussusception findings. A, Circumferential intimal tear (arrow) in proximal descending thoracic aorta. $\mathrm{B}$, Intimal flap inverted into abdominal aorta. Operative hinge point (arrowhead) was moved up, compared with CT finding due to reverse flow from femoral perfusion. C, Inverted flap was retrieved to thoracic aorta. Ant, Anterior; Post, posterior.

workup at the time. Chronic aortic pathology was an incidental finding on computed tomography (CT) during his imaging workup for pain. The CT angiogram demonstrated a descending thoracic aortic aneurysm from just below the left subclavian artery to the diaphragm level and localized abdominal aortic dissection without visualization of an intimal tear. Regular CT scan and antihypertensive medications were recommended, although his blood pressure was within normal range at 4 extremities. He visited our hospital 3 months later with ongoing pain that was more prominent on his abdomen. Despite no disease progression, follow-up CT angiogram indicated chronic dissection of the descending thoracic aorta above the diaphragm with aortic intimointimal intussusception into the abdominal aorta. The intimal flap was located in front of the ostia of the celiac and superior mesenteric arteries, causing dynamic obstruction of the celiac trunk and superior mesenteric artery (Figure 1). The 4.7-cm diameter of the descending thoracic aorta was not an indication for surgery; however, surgery was planned to alleviate symptoms and prevent ischemic injury to abdominal organs.

A left thoracotomy incision was made on the fifth intercostal space. Under femorofemoral bypass, a proximal clamp was placed between the left subclavian and the left common carotid arteries. The initial distal clamp on the mid-descending thoracic aorta was followed by an incision on the proximal descending thoracic aorta. The circumferential proximal tear site was identified just distal to the left subclavian artery, and proximal anastomosis was performed using a 20-mm woven vascular graft (Figure 2, A). Subsequently, distal perfusion was held and the crossclamp at the mid-thoracic aorta was released. The inverted flap was identified, retrieved into the thorax, and resected (Figure 2, $B$ and $C$ ). Distal anastomosis was made just proximal to the celiac trunk. The patient was extubated on the first postoperative day and discharged 2 weeks postsurgery without complications. CT angiogram performed 14 days postoperatively showed no leakage or pseudoaneurysm of the grafts and no ischemic change in abdominal organs. The patient's symptoms, such as flank and abdominal pain, disappeared, which supported the preoperative assessment of dynamic obstruction by the intimal flap.

\section{DISCUSSION}

Aortic intimointimal intussusception was first reported in 1962 by Hufnagel and Conrad. ${ }^{1}$ Most reported cases of aortic intimointimal intussusceptions are type A with the intimal tear starting just above the coronary artery ostia. ${ }^{2,3}$ Such aortic intimointimal intussusception could obstruct vessels from the aortic arch, resulting in neurologic dysfunction or blood pressure asymmetry in both arms. Aortic intimointimal intussusceptions in acute type B aortic dissection were also demonstrated in iatrogenic cases from catheter angiography or blunt trauma. ${ }^{4,5}$ As in type A aortic dissection, aortic intimointimal intussusception in type B dissection causes obstruction symptoms, including visceral or limb ischemia with occasional mortality.

Our case is characteristic in that it was manifested as a chronic course in type B aortic dissection and caused ischemic symptoms associated with obstruction of the visceral blood flow. To the best of our knowledge, this is the first case of aortic intimointimal intussusception presented in chronic type B aortic dissection. Previous back trauma possibly caused circumferential intimal tear on the proximal descending thoracic aorta and aortic intimointimal intussusception. The patient did not have a history of 
hypertension, and CT scan demonstrated no atherosclerosis throughout his aorta. There was no familial history of aortic pathology or connective tissue disease, which is why the trauma is the most suitable cause. Absence of a flap in the descending thoracic aorta caused misdiagnosis of an aortic aneurysm combined with abdominal aortic dissection on initial presentation.

\section{CONCLUSIONS}

We report aortic intimointimal intussusception in traumatic chronic type B aortic dissection with associated symptoms of visceral organ ischemia. This uncommon presentation of aortic dissection should be considered if the circumferential flap exists distal to the aneurysmal portion of the aorta.

\section{References}

1. Hufnagel CA, Conrad PW. Intimo-intimal intussusception in dissecting aneurysms. Am J Surg. 1962;103:727-31.

2. Debakey ME, Lawrie G. Intimal intussusception: unusual complication of dissecting aneurysm. J Vasc Surg. 1984;1:566-8.

3. Kastan DJ, Sharma RP, Keith F, Shetty PC, Burke MW. Intimo-intimal intussusception: an unusual presentation of aortic dissection. Am J Roentgenol. 1988; 151:603-4.

4. Mirick AL, Patel HJ, Deeb GM, Williams DM. Aortic intussusception complicating diagnostic angiography: recognition and management. Ann Thorac Surg. 2013;95:1776-8.

5. Kim H, Chung H, Choi J. Intimointimal intussusception in traumatic type B aortic dissection: CT and aortographic findings. Clin Radiol. 2000;55:487-8.

\title{
EDITORIAL COMMIENTARY
}

\section{An intimal cylinder in the descending aorta}

\author{
Yutaka Okita, MD
}

See related article on pages e33-5.

Intimointimal intussusception is a very rare complication of aortic dissection. Few cases have been reported in the English-language cardiology, cardiothoracic surgery, and radiology literature-only 30 or so since 1980 . Most reported cases have occurred in men, in a relatively younger group, aged 31 to 66 (mean: 51.8) years.

This uncommon variation usually occurs in acute type A dissections: the ascending aortic intima flaps around its circumference, detaches from the media, and forms a tubelike structure that may prolapse, either antegrade into the ascending aortic lumen, or retrograde into the left ventricular outflow tract and cavity. Usually, the site of the intimal tear is near the sinotubular junction of the ascending aorta. Although rarely reported, some such cases are complicated by chronic aortic dissection or Stanford type B dissection.

\footnotetext{
From the Division of Cardiovascular Surgery, Department of Surgery, Kobe University, Kobe, Japan.

Disclosures: Author has nothing to disclose with regard to commercial support.

Received for publication June 21, 2015; accepted for publication June 23, 2015; available ahead of print July 16, 2015.

Address for reprints: Yutaka Okita, MD, Division of Cardiovascular Surgery, Department of Surgery, 7-5-2, Kusunoki-cho, Chuo-ku, Kobe, Hyogo, Japan 650-0017

(E-mail: yokita@med.kobe-u.ac.jp).

J Thorac Cardiovasc Surg 2015;150:e35-6

$0022-5223 / \$ 36.00$

Copyright (c) 2015 by The American Association for Thoracic Surgery

http://dx.doi.org/10.1016/j.jtcvs.2015.06.043
}

Antegrade intussusceptions can partially or completely occlude the ostia of the branches of the distal or proximal aorta, and might cause dynamic obstruction malperfusion syndrome, such as coronary insufficiency, stroke, transient ischemic attack, paraplegia, or visceral or limb ischemia. Retrograde intussusceptions may severely impair

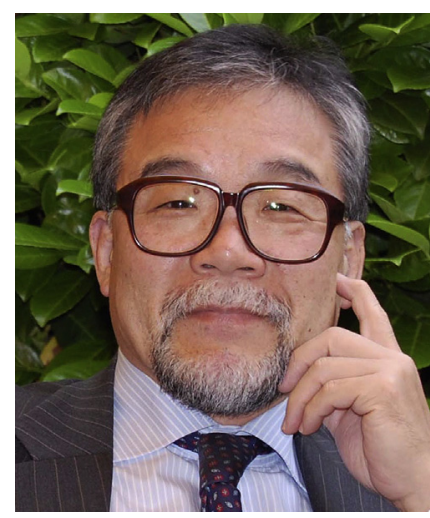
left ventricular filling in diastole, and can worsen aortic regurgitation, as well as produce occlusion of the coronary ostia and acute coronary ischemia.

This complication of aortic dissection seldom causes "static obstruction" of the aortic branches. Most cases have undergone emergent or elective surgery, and clinical results were satisfactory, which, of course, may have depended on patients' preoperative condition. We experienced the case of a 63-year-old man with acute type A aortic dissection with intimo-intimal intussusception, which caused severe aortic valve regurgitation and coronary ischemia. He was in a state of shock, and emergency hemiarch replacement was performed without any sequelae.

Dr Kim, Dr Ahn, and their associates at Seoul National University ${ }^{1}$ reported a very rare case of a man aged 38 years who had chronic type B aortic dissection with intimointimal 